\title{
Hughes-Stovin Syndrome or Vascular Behçet's Disease?
}

\author{
Silviu-Mihail Dumitru1, Antonela Dragomir ${ }^{1,2}$ and Florin Dumitru Mihaltan ${ }^{1,2}$ \\ ${ }^{1}$ Marius Nasta, National Institute of Pneumophtisiology, Romania \\ ${ }^{2}$ Carol Davila, University of Medicine and Pharmacy, Bucharest, Romania
}

\begin{abstract}
Hughes-Stovin Syndrome is a very rare pathology of unknown etiology with a lethal potential. It is characterized by the association of pulmonary artery aneurysms and peripheral venous thrombosis. We report the case of a 33 years old man with no prior illnesses, who came in for hemoptysis in small quantities. Based on the clinical presentation, the radiological studies and the histopathological finding we made our diagnosis of Hughes-Stovin Syndrome. The response to treatment was very good and at the 12 months follow-up the patient was completely asymptomatic, with no new radiological lessions on chest CT.
\end{abstract}

Hughes-Stovin syndrome (SHS) is a pathology of unknown etiology with a lethal potential. This syndrome was named in honor of two English doctors - Dr. John Patterson Hughes and Dr. Peter George Ingle Stovin. In 1959, the two doctors described for the first time the syndrome as the association between deep vein thrombosis and pulmonary segmental artery aneurysms [1].

SHS is an extremely rare pathology, with less than 40 cases published in the English medical literature. In 2007, an article was published in the journal " Clinical Rheumatology" the conclusion of which was that SHS shares common clinical, radiological and histopathological features with Behçet's disease [2], and the use of the term incomplete Behçet's disease is well warrant to describe SHS, although the same authors state in the article that the link between this two pathological entities is still speculative, as long as their etiology remains unknown. In 2004, in the journal "Clinical and experimental Rheumatology", a group of authors reached the same conclusion stating that the clinical, radiological and histopathological differences between the pulmonary involvement of these two pathologies are imperceptible [3].

In the framework of this article we present the case of a man age 33, nonsmoker, without exposure to respiratory hazards, former professional rugby player, without a significant past medical or family history, without treatment at that time, which had presented in our medical service for hemoptysis in small quantity, that began a month and a half before that moment, when he discharged about $50 \mathrm{ml}$ of blood, the next episode of hemoptysis occurred during the day in which he presented at the hospital with a discharge of about $60 \mathrm{ml}$ of blood. Whilst questioning the patient about other symptoms we found out the approximately a year ago the patient observed an increase in the volume of his right calf associated with pain, redness, an increase of local temperature, all of which resolved spontaneously within the next few days. It is important to remark that at moment of admission the patient denied chest pain, fever, dyspnea, arthralgia, rashes, bruises or weight loss. Furthermore we must specifically mention that the patient does not have a history of chronic pulmonary disease, immunosuppressive therapy or proximity to known tuberculosis patients. The physical examination at the time of the hospitalization did not reveal any notable elements. The complete blood count and biochemistry tests showed a biological inflammatory syndrome without leukocytosis; it is worth mentioning that multiple tests of the D-dimers were negative. A first tomographic examination showed a pulmonary consolidation process in the right inferior lobe with air bronchogram sign and minimal bilateral pleural fluid accumulation (Figure 1). A second tomographic examination, preformed 7 days later, revealed bilateral pulmonary consolidation with the same characteristics as the previous observed consolidation (Figure 2). Through serial bronchoscopic examinations, an evolution of the lesions was observed, at the first examination we observed rare bilateral anthracotic spots and an active hemorrhagic source at the level of the right basal pyramid (the precise source of the bleeding was not identified, just that the source could be a intersegmentary carina and consequently the hypothesis of bronchovascular fistula arouse), no other notable elements were identified. The latter bronchoscopic examination recorded the cessation of the bleeding (under the hemostatic effect of the medication), and without the constant stream of blood we identified an area of vascular ectasic hyperplasia at the right anterobazal carina. A hematology consult revealed a hereditary thrombophilia thru a mutation of the MTHFR gene - methyl tetrahididrofolat reductase (C677 mutation T A1298Cheterozygous genotype) and the mutation of the second coagulation factor - prothrombin - heterozygous genotype.

The hemorrhage could not be controlled by conservative maneuvers, and thus the indication for right inferior lobectomy arouse, under which the evolution was favorable, with the remission of hemoptysis.

The pathology exam of the resected specimen showed the following macroscopic findings (Figure 3): on the wall of the baso-dorsal segmentary brochi there was a $2-3 \mathrm{~mm}$ orifice, with subepithelial blood suffusion, which communicated with an aneurysmal dilation of the baso-dorsal arterial branch, this aneurysm had a long axis of about 15 $\mathrm{mm}$. The microscopic examination revealed a dilated arterial vessel with patchy fibrosis, lymphocyte infiltration of the media, parenchymal areas of remodeling with fibrosclerosis and small thrombosed arteriolar

*Correspondence to: Silviu-Mihail Dumitru MD, Marius Nasta, National Institute of Pneumophtisiology, Romania, E-mail: Dumitrusm@gmail.com

Key words: Hughes-Stovin Syndrome, Vascular Behçet's Disease.

Received: September 25, 2019; Accepted: September 31, 2019; Published: November 04, 2019 



Figure 1. A first tomographic examination showed a pulmonary consolidation proces in the right inferior lobe with air bronchogram sign and minimal bilateral pleural fluid accumulation
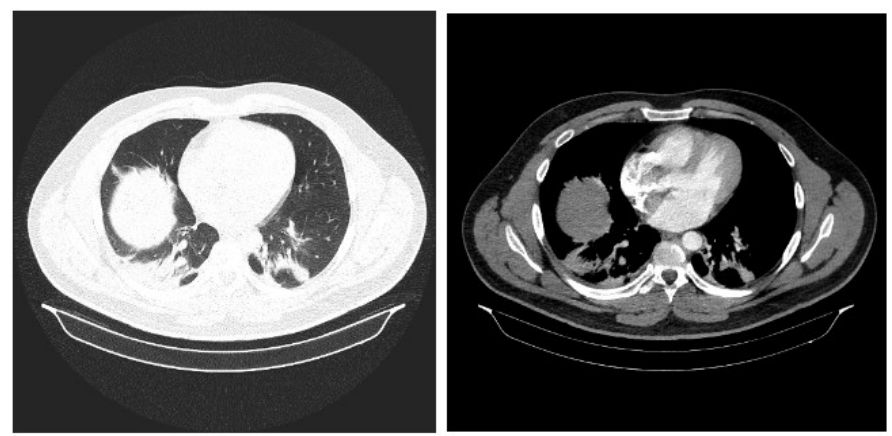

Figure 2. A second tomographic examination, preformed 7 days later, revealed bilateral pulmonary consolidation with the same characteristics as the previous observed consolidation

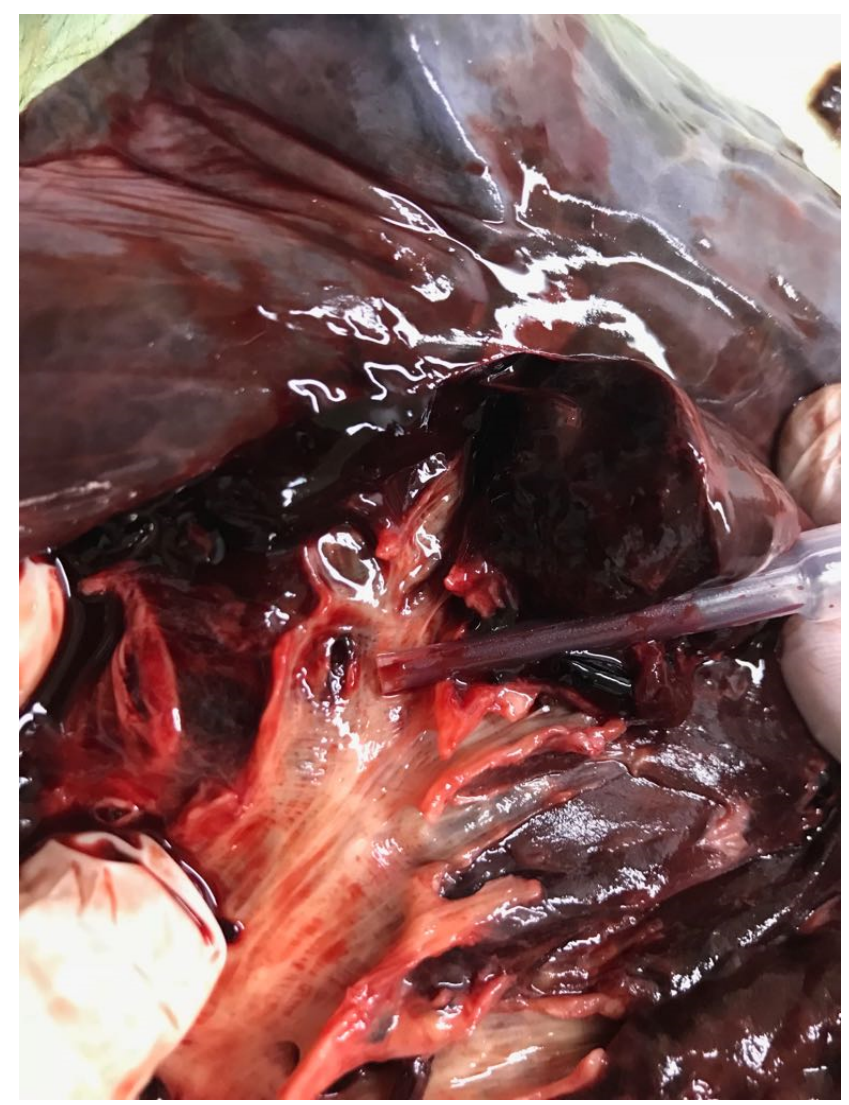

Figure 3. The pathology exam of the resected specimen showed the following macroscopic findings branches. Gathering the newly acquired data, the following diagnoses were made: postero-basal pulmonary aneurysm of the right lung with bronchovascular fistula and moderate hereditary thrombophilia with an episode of deep vein thrombosis.

After this diagnosis the patient was placed on oral anticoagulation, namely anti vitamin $\mathrm{k}$ for the hereditary thrombophilia and he was discharged. At 12 month follow-up the patient was completely asymptomatic, with no new radiological lessions on chest CT.

The clinical presentation of patients with SHS is heterogeneous, including cough, hemoptysis, dyspnea, signs and symptoms of deep vein thrombosis, chest pain, fever, chills, signs and symptoms of pulmonary hypertension or intracranial hypertension. At first glance, we would be tempted to organize these signs and symptoms into two categories: on the one hand those caused by pulmonary artery aneurysms, including those caused by their complications, and on the other hand, those caused by deep vein thrombosis and it's complications (eg pulmonary embolism, chronic post-thromboembolic pulmonary hypertension) but the grouping of the signs and symptoms in this manner seem to be forced and might not follow the pathophysiological pathway. Balci, et al. reported in "The Journal of Magnetic Resonance Imaging" the case of a patient with deep vein thrombosis and pulmonary thromboembolism in the context of SHS, which received a Greenfield filter, but the pulmonary thromboembolism was repeated [4], this fact is interpreted as a possible reason for pulmonary arterial thrombosis, in situ thrombosis. Thus without the intimate knowledge of this disease pathophysiology we cannot apply a scholastic template to the clinical presentation of SHS patients.

In the medical literature in the case of SHS the cause of the hypercoagulation state remains unknown, but there are reports of the association between hyperhomocysteinemia or prothrombin mutations and Behçet's disease [5]. In our patient, hyperhomocysteinemia enters the scene through the mutation of the MTHFR gene, responsible for the synthesis of methyltetrahydrofolate reductase, a catalytic enzyme in the methylation cycle.

In the differential diagnosis between Behçet's disease and HughesStovin's syndrome, we started with the diagnostic criteria of Behçet's: major criteria - recurrent oral ulcers objectified by a doctor (three episodes in the last year) and minor criteria - recurrent genital ulcers or fibrotic lesions objectified by to a physician, anterior or posterior uveitis, cells in the vitreous body or retinal vasculitis objectified by an ophthalmologist, skin lesions (erythema nodes, pseudofolliculitis, papulopustular lesions) and the behcetin / pathergy test. Starting from the absence of oral ulceration during the hospitalization period, as well as in the medical history and admitting that this is a sine qua non criterion in the diagnosis of Behçet's disease, we conclude that it cannot be the classic form of Bechet's disease.

Regarding the treatment cited in the literature, we can speak of two currents, one conservative, which is based on immunosuppression to limit aneurysmal growth (glucocorticoids, cyclophosphamide, azathioprine or cyclosporine A) and a radical one, which advocates for the resection of vascular defects [6]. Anticoagulant therapy is added to both of these approaches. Oral anticoagulation is recommended to reduce the risk of deep vein thrombosis and pulmonary thrombembolism, but this recommendation only applies to patients who have had a medical or surgical intervention through which the aneurysmal lesions were controlled.

It is utopic to consider that a disease with such a low prevalence will in the future have the attention of the medical community, so that the etiological and pathophysiological enigmas can be resolved, but an 
aggregation of the data related to these patients might shed light on the most important question for patients, namely: which is the safest and most effective treatment?

\section{References}

1. Hughes JP, Stovin PG (1959) Segmental pulmonary artery aneurysms with peripheral venous thrombosis. Br J Dis Chest 53: 19-27. [Crossref]

2. Yasser Emad, Yasser Ragab, El-Marakbi A, Saad A, Osama Ibrahim, et al. (2019) A case of Hughes-Stovin syndrome (incomplete Behçet's disease) with extensive arterial involvement: Unmasking the true face of a rare syndrome. Z Rheumatol 78: 365-371.
3. Erkan D, Yazici Y, Sanders A, Trost D, Yazici H (2004) Is Hughes-Stovin syndrome Behçet's disease? Clin Exp Rheumatol 22: S64-68. [Crossref]

4. Cem Balci N, Richard C Semelka, Tara C Noone, Suvipapun Worawattanakul (2001) Multiple Pulmonary Aneurysms Secondary to HugheslStovin Syndrome: Demonstration by MR Angiography.

5. Leiba M, Sidi Y, Gur H, Leiba A, Ehrenfeld M (2001) BehÃßet's disease and thrombophilia. Ann Rheum Dis 60: 1081-1085.

6. Mohammed Abdelbary, Ahmed El-Masry, Motaz S Rabiec (2016) Life threatening hemoptysis from Hughes Stovin syndrome: Is it that rare? Respir Med Case Rep 19: 98-102.

Copyright: ( 2019 Dumitru SM. This is an open-access article distributed under the terms of the Creative Commons Attribution License, which permits unrestricted use, distribution, and reproduction in any medium, provided the original author and source are credited. 